\title{
Markers of memory CD8 T cells depicting the effect of the BNT162b2 mRNA COVID-19 vaccine in Japan
}

\section{Hiroyuki Kondo}

University of Tokushima

\section{Takahiro Kageyama}

Chiba University

\section{Shigeru Tanaka}

Graduate School of Medicine, Chiba University https://orcid.org/0000-0002-8466-110X

\section{Shin-ichi Tsukumo}

University of Tokushima

Kunihiro Otsuka

University of Tokushima

\section{Yoichi Mashimo}

Graduate School of Medicine, Chiba University

\section{Yoshihiro Onouchi}

Department of Public Health, Chiba University Graduate School of Medicine https://orcid.org/00000003-1267-5913

\section{Hiroshi Nakajima}

https://orcid.org/0000-0001-8595-9381

Koji Yasutomo ( $\square$ yasutomo@tokushima-u.ac.jp)

University of Tokushima https://orcid.org/0000-0003-0411-2037

\section{Article}

Keywords: COVID-19 vaccine, BNT162b2, mRNA vaccines, memory CD8+ T cells

Posted Date: October 8th, 2021

DOI: https://doi.org/10.21203/rs.3.rs-936363/v1

License: (c) (i) This work is licensed under a Creative Commons Attribution 4.0 International License. Read Full License 
Markers of memory CD8 $\mathrm{T}$ cells depicting the effect of the BNT162b2 mRNA

COVID-19 vaccine in Japan

by

Hiroyuki Kondo', Takahiro Kageyama ${ }^{2}$, Shigeru Tanaka², Kunihiro Otsuka ${ }^{1}$, Shin-ichi Tsukumo ${ }^{1}$, Yoichi Mashimo ${ }^{3}$, Yoshihiro Onouchi ${ }^{3}$, Hiroshi Nakajima ${ }^{2}$, Koji Yasutomo ${ }^{1,4,5,6}$

1. Department of Immunology \& Parasitology, Graduate School of Medicine, Tokushima University, Tokushima, Japan

2. Department of Allergy and Clinical Immunology, Graduate School of Medicine, Chiba University, Chiba, Japan

3. Department of Public Health, Graduate School of Medicine, Chiba University, Chiba, Japan

4. Department of Interdisciplinary Researches for Medicine and Photonics, Institute of Post-LED Photonics, Tokushima University, Tokushima, Japan

5. The Research Cluster Program on Immunological diseases, Tokushima University, Tokushima, Japan

6. Tokushima International Science Institute (TISI), Tokushima University, Tokushima, Japan

Address correspondence to:

Hiroshi Nakajima, M.D., Ph.D.

Department of Allergy and Clinical Immunology, Graduate School of Medicine, Chiba University, 1-8-1 Inohana, Chiba City, Chiba 260-8670, Japan

Tel: $+\mathbf{8 1 - 4 3 - 2 2 6 - 2 1 9 8}$

Fax: +81-43-226-2199

E-mail: nakajimh@faculty.chiba-u.jp

or

Koji Yasutomo, M.D., Ph.D.

Department of Immunology \& Parasitology, Graduate School of Medicine, Tokushima University, 3-18-15 Kuramoto, Tokushima 770-8503, Japan

Tel.: +81-88-633-7048

Fax: +81-88-633-7114

E-mail: yasutomo@tokushima-u.ac.jp 


\begin{abstract}
BNT162b2, a nucleoside-modified mRNA vaccine for SARS-CoV-2 spike glycoprotein (S), provides approximately 95\% efficacy for preventing COVID-19. However, it remains unclear how effectively memory CD8+ $T$ cells are generated and which genetic and environmental factors affect the generation and function of memory CD8 + $\mathrm{T}$ cells elicited by this vaccine. Here, we investigated the frequency and functions of memory CD8+ T cells 3 weeks after the second vaccination in the Japanese population. Using a peptide-MHC pentamer, we detected an increased number of memory CD8+ $T$ cells in females compared with that in males, but the frequency of pentamer-positive cells was not correlated with antibody titers. Memory precursor effector cells (KLRG1-CD127+) among both CD8+ cells and pentamer+ cells and effector cells (CD38-HLA-DR+) among pentamer+ cells were more abundant in females than in males. Upon $S$ protein-mediated stimulation of $T$ cells, the intensity of CD107a and granzyme $B$ expression was increased in females compared with that in males, indicating stronger memory $\mathrm{CD8}+\mathrm{T}$ cell responses in females than in males. Our studies showed that the BNT162b2 vaccine elicits increased memory CD8+ $T$ cell proliferation and secondary $C T L$ responses in females compared with those in males in the Japanese population. These findings provide an important basis for the distinct sex difference in cellular immune responses to mRNA vaccination and suggest that memory precursor effector cells can be a simple marker to evaluate and boost cellular immunity induced by BNT162b2.
\end{abstract}




\section{Introduction}

Infection with severe acute respiratory syndrome coronavirus 2 (SARS-CoV-2) occurred in late 2019 and rapidly spread worldwide ${ }^{12}$. The disease (coronavirus disease 2019, COVID-19) has caused significant morbidity and mortality, with the emergence of viruses with various mutations in the spike glycoprotein. Worldwide, various attempts have been applied to develop novel vaccines and therapeutic strategies together with assessing immune responses against SARS-CoV-2 ${ }^{4}$.

The S protein of SARS-CoV-2 is needed for the entry of virus into the cells through interaction with angiotensin-converting enzyme II ${ }^{6}$. The S protein includes a signal peptide, an $\mathrm{S} 1$ protease fragment that contains a receptor-binding domain and an $\mathrm{S} 2$ protease fragment. By expecting the generation of memory $\mathrm{T}$ and $\mathrm{B}$ cells against the $S$ protein, BioNTech and Pfizer launched a new type of mRNA vaccine that encodes the SARS-CoV-2 full-length spike with modification by two proline mutations. Clinical trials of the vaccine revealed approximately $95 \%$ efficacy for protecting against COVID $-19^{78}$. The vaccine increased the serum levels of neutralizing antibodies against the S protein and IFN- $\gamma$ production from CD4+ and CD8+ T cells ${ }^{9}$. The generation of $\mathrm{T}$ cell memory after vaccination is required for long-term protective immunity to SARS$\mathrm{CoV}-2$. Indeed, one paper showed that memory $\mathrm{T}$ cells contribute to protection against SARS-CoV-2 rechallenge in a rhesus macaque model ${ }^{10}$. However, it remains unclear which environmental factors affect the generation of memory $\mathrm{T}$ cells, which are markers for memory $\mathrm{T}$ cells and how long protective immunity is maintained.

To this end, we tested the frequency and function of $\mathrm{CD} 8+\mathrm{T}$ cell responses against the S protein of SARS-CoV-2 before and three weeks after the second dose of the BNT162b2 mRNA vaccine in the Japanese population. We found an increased number of memory CD8 $+\mathrm{T}$ cells in females compared with that in males, but the frequency of S protein-specific CD8 + T cells was not correlated with antibody titers. The numbers of total and antigen-specific memory precursor effector cells (KLRG1$\mathrm{CD} 127+\mathrm{CD} 8+)$ and antigen-specific effector cells (CD38-HLA-DR+CD8+) were increased in females compared those in with males. These findings provide an important basis for the distinct sex differences in the cellular immune responses to mRNA vaccines and suggest that memory precursor effector cells can serve as markers to boost vaccine effectiveness. 


\section{Results}

Study design

We recruited 996 donors at Chiba University who received 2 rounds of administration of BNT162b2, a nucleoside-modified mRNA vaccine (Fig. 1A) ${ }^{11}$. Approximately $10 \%$ of Japanese individuals carry HLA-A*0201 ${ }^{12}$, and several epitopes of SARS-CoV-2 on HLA-A*0201 have been reported ${ }^{9}$. Thus, we first screened donors who carry HLA$A^{*} 0201$ to detect $S$ protein-specific T cells with pentamers composed of epitopes of SAR-CoV-2 and HLA-A*0201 (Fig. 1B). We amplified genomic DNA with specific primers for HLA-A*2 and A*0201 and found 118 donors carrying the HLA-A*0201 gene (Fig. B). The donors included 68 females and 50 males, and the ages ranged from 20 to 70 (Fig. 1C). The antibody titer against the S-protein was higher in females than in males, but the titer was not correlated with age in 118 donors, although studies using all 996 donors have shown an inverse correlation between antibody titer and age ${ }^{11}$.

More antigen-specific CD8 T cells were observed in females than in males after the second vaccination.

We selected the SARS-CoV-2 $\mathrm{S}_{269}$ (YLQPRTFLL) pentamer to detect antigenspecific CD8 T cells in our study because, among several pentamers for HLA-A*0201, this pentamer is the most frequently able to detect antigen-specific $\mathrm{T}$ cells from COVID-19 patients ${ }^{13}$. We finally tested SARS-CoV-2 S 269 (YLQPRTFLL) pentamer ${ }^{+}$ cells of 20 donors from the 118 donors (10 female and 10 male). We examined the phenotypes of SARS-CoV-2 $\mathrm{S}_{269}$ MHC class I pentamer ${ }^{+} \mathrm{CD}^{+} \mathrm{T}$ cells in PBMCs one week before the $1^{\text {st }}$ vaccination and three weeks after the second vaccination. The total number of $\mathrm{CD}^{+} \mathrm{T}$ cells among $\mathrm{CD}^{+}$cells was not different before and after vaccination (Fig. 2A and B). The pentamer ${ }^{+}$cells among CD8+ T cells were not detected before vaccination in any samples, but the pentamer ${ }^{+}$cells were detected 3 weeks after the second vaccination (Fig. $2 \mathrm{C}$ and D). The frequency of pentamer ${ }^{+}$cells 3 weeks after the $2^{\text {nd }}$ vaccination was significantly higher in females than in males (Fig. 2C and D). The antibody titer and frequency (Fig. 2E) or number (Fig. 2F) of pentamer ${ }^{+}$ cells were not correlated.

Increased numbers of memory precursors of CD8+T cells in females compared with those in males

We next evaluated the expression patterns of cell surface markers related to effector or memory functions on pentamer ${ }^{+} \mathrm{CD} 8+\mathrm{T}$ cells. We determined similar proportions of naïve or stem-like memory $\mathrm{T}$ cells $\left(\mathrm{CCR} 7^{+} \mathrm{CD} 45 \mathrm{RA}{ }^{+}\right)$, central memory 
cells $\left(\mathrm{CCR}^{+} \mathrm{CD}^{2} 5 \mathrm{RA}^{-}\right)$, effector memory cells $\left(\mathrm{CCR} 7^{-} \mathrm{CD}^{-} 5 \mathrm{RA}^{-}\right)$and terminally differentiated effector cells $\left(\mathrm{CCR} 7^{-} \mathrm{CD} 45 \mathrm{RA}^{+}\right)$in the $\mathrm{CD} 8^{+}$population between females and males before and after the second vaccination (Fig. 3A and B). The frequency of each proportion of total CD8 $+\mathrm{T}$ cells after the second vaccination was equivalent to that before vaccination. We then evaluated the frequency of each population among pentamer $^{+} \mathrm{CD} 8+\mathrm{T}$ cells after the second vaccination. Approximately $30 \%$ of pentamer ${ }^{+}$ cells exhibited an effector memory $\left(\mathrm{CCR}^{-} \mathrm{CD}^{-} \mathrm{RA}^{-}\right)$phenotype, and $\sim 30 \%$ or $10 \%$ of pentamer ${ }^{+}$cells were terminally differentiated effector $\left(\mathrm{CCR} 7^{-} \mathrm{CD} 45 \mathrm{RA}^{+}\right)$cells or naïve/stem-like memory T cells $\left(\mathrm{CCR} 7^{+} \mathrm{CD} 45 \mathrm{RA}{ }^{+}\right)$, respectively. The frequency of those three populations in females was equivalent to that in males (Fig. 3C and D).

The effector functions and memory precursors of CD8+ T cells are also distinguished by the expression patterns of CD127 and KLRG1 on CD8+ T cells ${ }^{14}$. We found more $\mathrm{KLRG1}{ }^{-} \mathrm{CD} 127^{+}$cells, memory precursor effector cells, among total CD8+ $\mathrm{T}$ cells in females than in males, but the numbers of other types of cells (KLRG1CD127-, KLRG1 ${ }^{+} \mathrm{CD} 127^{+}, \mathrm{KLRG}^{+} \mathrm{CD} 127^{-}$) were comparable between males and females (Fig. 3E and F). Similar to the data regarding total CD8+ T cells, the frequency of KLRG1 ${ }^{-} \mathrm{CD} 127^{+}$cells among pentamer ${ }^{+} \mathrm{CD} 8 \mathrm{~T}$ cells was higher in females than in males (Fig. 3G and H). The frequency of $\mathrm{KLRG}^{-} \mathrm{CD} 127^{+}$cells among pentamer ${ }^{+} \mathrm{CD} 8+$ $\mathrm{T}$ cells was not correlated with the frequency of pentamer ${ }^{+}$cells (Fig. 3I). These data suggest that $\mathrm{KLRG1}{ }^{-} \mathrm{CD} 127^{+}$cells are a simple marker to evaluate cellular immunity after BNT162b2 mRNA vaccination.

Increased memory/effector CD8+T cell population in females compared with that in males

The expression of CD28 and PD-1 can be useful in distinguishing the activation status of $\mathrm{CD} 8+\mathrm{T}$ cells. The frequency of $\mathrm{CD} 28^{+}$cells among total $\mathrm{CD} 8+\mathrm{T}$ cells (Fig. 4A and B) was equivalent to that among pentamer ${ }^{+}$cells after the second vaccination (Fig. 4C and D). The expression of PD-1 in pentamer ${ }^{+} \mathrm{CD} 8+\mathrm{T}$ cells was increased compared with that in total CD8+ T cells, and the frequency of PD- $1^{+}$cells among pentamer ${ }^{+} \mathrm{CD} 8+\mathrm{T}$ cells was comparable between females and males.

CD8+ T cells can also be classified by the level of activation with HLA-DR and CD38 expression patterns. Coexpression of CD38 and HLA-DR defines early activation status, with high effector functions and higher susceptibility to cell death after their function has been accomplished. CD38-HLA-DR ${ }^{+}$CD8 + T cells exhibit an elevated functional response with an increased survival rate during viral infection ${ }^{15}$. Four populations based on the expression pattern of CD38 and HLA-DR were detected 
in pentamer ${ }^{+}$cells. Although the frequency of CD38 ${ }^{+} \mathrm{HLA}^{-\mathrm{DR}^{+}}$or CD38-HLA-DR ${ }^{+}$ cells among total CD8+ T cells was comparable between females and males (Fig. 4E and $\mathrm{F}$ ), we found a similar frequency of CD38 ${ }^{+} \mathrm{HLA}^{-\mathrm{DR}^{+}}$cells in females and males and a higher frequency of CD38-HLA-DR ${ }^{+} \mathrm{CD} 8+\mathrm{T}$ cells among pentamer ${ }^{+} \mathrm{CD} 8+\mathrm{T}$ cells in females than in males (Fig. 4G and H). The number of CD38-HLA-DR ${ }^{+}$cells among pentamer ${ }^{+} \mathrm{CD} 8+\mathrm{T}$ cells was correlated with the frequency of pentamer ${ }^{+}$cells (Fig. 4I). These data suggest that females are able to generate higher functional responses by the second dose of BNT162b2.

\section{Memory $T$ cell responses}

We next tested the epitope-specific effector functions of CD8 + T cells by stimulating PBMCs with an S protein-derived peptide for 14 days. We tested the frequency of antigen-specific T cells, cytotoxic functions and cytokine secretion levels. The stimulation of $\mathrm{T}$ cells with the peptides led to the expansion of T cells, and the frequency of pentamer ${ }^{+} \mathrm{CD} 8+\mathrm{T}$ cells was greater in females than in males (Fig. 5A). The killing capability of pentamer ${ }^{+} \mathrm{CD} 8+\mathrm{T}$ cells was assessed by staining cell surface CD107a, a marker for degranulation, before and after T cell stimulation. Approximately $40 \%$ of pentamer ${ }^{+} \mathrm{T}$ cells expressed CD107a before stimulation, and the frequency was similar between males and females. Upon stimulation with the peptide, more than $70 \%$ of pentamer ${ }^{+}$cells expressed CD107a, and the frequency was higher in females than in males (Fig. 5B). The intensity of CD107a staining tended to be higher in females than in males.

The concentrations of molecules secreted from T cells were measured 14 days after $\mathrm{T}$ cell stimulation. IFN- $\gamma$ and TNF- $\alpha$ tended to be more highly secreted in cells from females than in cells from males, but the levels were comparable between females and males (Fig. 5C). Only granzyme B was secreted from $\mathrm{T}$ cells in greater amounts by females than by males in this study, suggesting stronger cytotoxic responses in females than males during the memory phase after the second COVID-19 vaccination.

\section{Discussion}

The adaptive immune system is essential for preventing or clearing the virus in the body 16171819 . Vaccines against virus infection activate both innate and adaptive immunity by mimicking natural infections ${ }^{1620}$. Antibodies are crucial for preventing the entry of free virus into cells, and the vaccine effect is easily determined by measuring the antibody titer in the serum. In contrast, although $\mathrm{T}$ cells are needed to clear infected cells, the measurement of antigen-specific $\mathrm{T}$ cells is not easy because HLA typing and 
determination of the antigen epitope is required to detect the T cells. Here, we investigated the frequency, cell surface markers and function of antigen-specific CD8 T cells from donors who received a second BNT162b2 mRNA vaccine. In the present study, we demonstrated that SARS-CoV-2-specific memory $\mathrm{T}$ cell responses were more abundant in females than in males. Furthermore, we found that $\mathrm{CD} 127^{+} \mathrm{KLRG}^{-}$cells could be used as indicators of memory CD8+ T cell generation by BNT162b2 vaccination.

Our previous study showed a higher titer of anti-S protein antibodies in females than in males ${ }^{11}$. Furthermore, we detected a higher number of antigen-specific CD8+ T cells in females than in males. How can the sex difference in the immune response against BNT162b2 mRNA vaccine in the Japanese population be explained? The involvement of body weight would be unlikely because we did not observe a positive correlation between BMI and the frequency of antigen-specific T cells or antibody titer in this study ${ }^{11}$. The most likely explanation is distinct immune responses against the vaccine between females and males, at least in the Japanese population. Previous studies have also shown stronger immune responses in females than in males after vaccination against viruses, including influenza ${ }^{21}$. Future studies on detailed molecular signatures in pentamer ${ }^{+} \mathrm{T}$ cells in female and male donors could clarify the differential cellular immune responses against BNT162b2 vaccination.

By testing several cell surface markers that distinguish subsets of memory and effector T cells, we detected an increased frequency of $\mathrm{CD} 8^{+} \mathrm{KLRG} 1^{-} \mathrm{CD} 127^{+}$cells in females compared with that in males. The same tendency was observed for pentamer+ cells. As KLRG1-CD $127^{+}$cells are defined as memory precursor effector cells that display an increased ability to form long-lived memory cells and short-lived effector cells ${ }^{22}$, female donors should have better potential to generate memory CD8+ T cells. As we still do not have many COVID-19 cases even after vaccination, we are not able to assess whether female donors are more resistant to SARS-CoV-2 after a second dose of vaccination. Nevertheless, upon in vitro stimulation of CD8 T cells with peptides, there was higher expression of cytotoxicity-related molecules in female donors than in male donors, suggesting stronger memory $\mathrm{T}$ cell responses in females than in males after the second vaccination.

CD8+ T cells from convalescent subjects have been found to lack expression of the CD38 and HLA-DR markers, suggesting suboptimal priming of CD8+ T cells in primary COVID-19 ${ }^{23}$. In contrast to the case for natural infection with SARS-CoV-2, CD $38^{+}$HLA-DR $^{+}$pentamer ${ }^{+} \mathrm{T}$ cells were detected 21 days after the second vaccination, and the frequency was higher in females than in males, suggesting that this population 
would also be an indicator for the generation of memory CD8+ T cells. Regarding the distinct expression patterns of CD38 and HLA-DR between natural infection and vaccination and since impairment of dendritic functions in patients with COVID-19 has been reported ${ }^{24}$, the lack of impairment of dendritic cells might lead to strong stimulation of CD8+ T cells by vaccination.

Polyclonal T cells are crucial for clearing virus infection, and the generation of polyfunctional memory $\mathrm{T}$ cells by vaccination is required for conferring protective immunity. Here, we tested memory CD8+ T cells against only one epitope on HLA$A^{*} 0201$. Therefore, it would be necessary to evaluate the frequency and functions of CD8 + T cells across a broad range of HLA class I alleles and SARS-CoV-2 peptides to understand a more detailed landscape of CD8 $+\mathrm{T}$ cell responses in COVID-19 in future studies. However, even when evaluating total CD8+ T cells, we detected increased numbers of KLRG1 ${ }^{-} \mathrm{CD} 127^{+}$cells in females compared with those in males, strongly suggesting that $\mathrm{CD} 8^{+} \mathrm{KLRG} 1^{-} \mathrm{CD} 127^{+}$cells would be a useful indicator for evaluating the generation of memory CD $8+\mathrm{T}$ cells by BNT162b2 mRNA vaccination without using pentamer staining. 


\section{Acknowledgments}

This study is supported by JST Moonshot R\&D grant numbers JPMJMS2025 and a funding from Tokushima University - Technion - Nichia Corporation collaboration. 


\section{Methods}

\section{Patient recruitment}

Healthcare workers in Chiba University Hospital who received the BNT162b2 mRNA COVID-19 vaccine (Pfizer, Inc., and BioNTech) were recruited. Blood samples were collected 0-2 weeks before the $1^{\text {st }}$ dose and 3 weeks after the $2^{\text {nd }}$ dose. Antibody responses were analyzed using Elecsys ${ }^{\circledR}$ Anti-SARS-CoV-2S on the Cobas 8000 e801 module (Roche Diagnostics, Rotkreuz, Switzerland). Background information was collected ${ }^{11}$, and all participants gave written informed consent before undergoing any study procedures. This study was approved by the ethical committee for medical research involving human subjects at Chiba University and Tokushima University.

\section{PBMC isolation}

PMBCs were isolated from peripheral blood and stored in liquid nitrogen ${ }^{11}$. Frozen PMBCs were transported from Chiba University to Tokushima University and stored in liquid nitrogen. Stored PMBCs were thawed and immediately used in various experiments.

\section{HLA screening}

Genomic DNA was extracted from white blood cells and amplified with primers for HLA-A*02, forward primer 5'- CACTCCTCGTCCCCAGGCTGT -3' and reverse primer 5'- CGTGGCCCCTGGTACCCGT-3', and HLA-A*0201, forward primer 5'TCCTCGTC

CCCAGGCTCT -3' and reverse primer 5'- GTGGCCCCTGGTACCCGT-3', with a thermal cycler (TaKaRa).

\section{Flow cytometry}

Cryopreserved PBMCs were thawed and stained with APC-labeled HLA-A*0201 pentamers (YLQPRTFLL: SARS-CoV-2 $\mathrm{S}_{269}$ ) and antibodies. For pentamer staining, the cells were incubated for $15 \mathrm{~min}$ at room temperature, washed, and then stained with fluorochrome-conjugated antibodies for specific surface markers for $20 \mathrm{~min}$ at $4^{\circ} \mathrm{C}$. The antibody mixture consisted of anti-human CD3 (Biolegend, clone HIT3a), CD8 $\alpha$ (Biolegend, clone HIT8a), CCR7 (Biolegend, clone G043H7), CD45RA (Biolegend, clone HI100), KLRG1 (Biolegend, clone SA231A2), CD127 (Biolegend, clone A019D5), CD28 (Biolegend, clone CD28.2), PD-1 (Biolegend, clone EH12.2H7), CD38 (Biolegend, clone HIT2), HLA-DR (Biolegend, clone L234), and CD107a 
(Biolegend, clone H4A3). After three additional washes with FACS buffer, the cells were analyzed on a FACSCanto II (BD Biosciences).

\section{T cell stimulation}

Cryopreserved PBMCs $\left(5 \times 10^{5}\right.$ cells $)$ were thawed and stimulated in the presence of a peptide (YLQPRTFLL, $10 \mu \mathrm{g} / \mathrm{ml}$ ) for 14 days using a U-bottom 96-well plate.

\section{Cytokine measurement}

Cytokine concentrations were measured using the LEGENDplex ${ }^{\mathrm{TM}}$ Human CD8/NK Panel (13-plex) with a Filter Plate (Biolegend). In brief, we incubated cell culture supernatant with the human $\mathrm{CD} 8 / \mathrm{NK}$ panel premixed beads on a plate shaker. After incubation, human $\mathrm{CD} 8 / \mathrm{NK}$ panel detection antibodies were added to the mixed solution and incubated on a plate shaker. After incubation, the mixed solution was analyzed on a FACSCanto II (BD Biosciences).

\section{Statistical analysis}

The Mann-Whitney U test or Wilcoxon signed-rank test was used to compare data between two unpaired groups or between two paired groups, respectively. The Spearman correlation test was used to assess the significance of the correlation. 


\section{References}

1. Zhou P, Yang XL, Wang XG, Hu B, Zhang L, Zhang W, et al. A pneumonia outbreak associated with a new coronavirus of probable bat origin. Nature 2020, 579(7798): 270-273.

2. Zhu N, Zhang D, Wang W, Li X, Yang B, Song J, et al. A Novel Coronavirus from Patients with Pneumonia in China, 2019. N Engl J Med 2020, 382(8): 727733.

3. Huang C, Wang Y, Li X, Ren L, Zhao J, Hu Y, et al. Clinical features of patients infected with 2019 novel coronavirus in Wuhan, China. Lancet 2020, 395(10223): 497-506.

4. Krause PR, Fleming TR, Longini IM, Peto R, Briand S, Heymann DL, et al. SARS-CoV-2 Variants and Vaccines. N Engl J Med 2021, 385(2): 179-186.

5. Tregoning JS, Flight KE, Higham SL, Wang Z, Pierce BF. Progress of the COVID-19 vaccine effort: viruses, vaccines and variants versus efficacy, effectiveness and escape. Nat Rev Immunol 2021.

6. Mariano G, Farthing RJ, Lale-Farjat SLM, Bergeron JRC. Structural Characterization of SARS-CoV-2: Where We Are, and Where We Need to Be. Front Mol Biosci 2020, 7: 605236.

7. Polack FP, Thomas SJ, Kitchin N, Absalon J, Gurtman A, Lockhart S, et al. Safety and Efficacy of the BNT162b2 mRNA Covid-19 Vaccine. N Engl J Med 2020, 383(27): 2603-2615.

8. Walsh EE, Frenck RW, Jr., Falsey AR, Kitchin N, Absalon J, Gurtman A, et al. Safety and Immunogenicity of Two RNA-Based Covid-19 Vaccine Candidates. N Engl J Med 2020, 383(25): 2439-2450.

9. Sahin U, Muik A, Vogler I, Derhovanessian E, Kranz LM, Vormehr M, et al. BNT162b2 vaccine induces neutralizing antibodies and poly-specific T cells in 
humans. Nature 2021, 595(7868): 572-577.

10. McMahan K, Yu J, Mercado NB, Loos C, Tostanoski LH, Chandrashekar A, et al. Correlates of protection against SARS-CoV-2 in rhesus macaques. Nature 2021, 590(7847): 630-634.

11. Kageyama T, Ikeda K, Tanaka S, Taniguchi T, Igari H, Onouchi Y, et al. Antibody responses to BNT162b2 mRNA COVID-19 vaccine and their predictors among healthcare workers in a tertiary referral hospital in Japan. Clin Microbiol Infect 2021.

12. Ikeda N, Kojima H, Nishikawa M, Hayashi K, Futagami T, Tsujino T, et al. Determination of HLA-A, -C, -B, -DRB1 allele and haplotype frequency in Japanese population based on family study. Tissue Antigens 2015, 85(4): 252259.

13. Rha MS, Jeong HW, Ko JH, Choi SJ, Seo IH, Lee JS, et al. PD-1-Expressing SARS-CoV-2-Specific CD8(+) T Cells Are Not Exhausted, but Functional in Patients with COVID-19. Immunity 2021, 54(1): 44-52 e43.

14. Chang JT, Wherry EJ, Goldrath AW. Molecular regulation of effector and memory T cell differentiation. Nat Immunol 2014, 15(12): 1104-1115.

15. Saez-Cirion A, Lacabaratz C, Lambotte O, Versmisse P, Urrutia A, Boufassa F, et al. HIV controllers exhibit potent CD8 T cell capacity to suppress HIV infection ex vivo and peculiar cytotoxic $\mathrm{T}$ lymphocyte activation phenotype. Proc Natl Acad Sci U S A 2007, 104(16): 6776-6781.

16. Ngono AE, Shresta S. Immune Response to Dengue and Zika. Annu Rev Immunol 2018, 36: 279-308.

17. Chow KT, Gale M, Jr., Loo YM. RIG-I and Other RNA Sensors in Antiviral Immunity. Annu Rev Immunol 2018, 36: 667-694.

18. St John AL, Rathore APS. Adaptive immune responses to primary and secondary dengue virus infections. Nat Rev Immunol 2019, 19(4): 218-230. 
19. Berry R, Watson GM, Jonjic S, Degli-Esposti MA, Rossjohn J. Modulation of innate and adaptive immunity by cytomegaloviruses. Nat Rev Immunol 2020, 20(2): 113-127.

20. Sadarangani M, Marchant A, Kollmann TR. Immunological mechanisms of vaccine-induced protection against COVID-19 in humans. Nat Rev Immunol 2021, 21(8): 475-484.

21. Klein SL, Flanagan KL. Sex differences in immune responses. Nat Rev Immunol 2016, 16(10): 626-638.

22. Martin MD, Badovinac VP. Defining Memory CD8 T Cell. Front Immunol 2018, 9: 2692.

23. Habel JR, Nguyen THO, van de Sandt CE, Juno JA, Chaurasia P, Wragg K, et al. Suboptimal SARS-CoV-2-specific CD8(+) T cell response associated with the prominent HLA-A*02:01 phenotype. Proc Natl Acad Sci U S A 2020, 117(39): 24384-24391.

24. Zhou R, To KK, Wong YC, Liu L, Zhou B, Li X, et al. Acute SARS-CoV-2 Infection Impairs Dendritic Cell and T Cell Responses. Immunity 2020, 53(4): 864-877 e865. 


\section{Figure legends}

\section{Figure 1}

(A) Study scheme for the collection of PBMCs from individuals administered the BNT162b2 vaccine. (B) Genotyping PCR analysis of genomic DNA from human white blood cells. Gel electrophoresis of the amplified HLA-A*02 fragment (854 bp) and HLA-*0201 fragment (813 bp). (C) Frequencies of sex and age of subjects that provided HLA-A* $0201^{+}$samples. (D) Antibody titers of males and females in HLA$A^{*} 0201^{+}$samples of after vaccination. (E) Scatter plots of antibody titer and cell number of HLA-A* $0201^{+}$samples after vaccination. ${ }^{* *}, \mathrm{p}<0.01$

\section{Figure 2}

(A) Flow cytometric analysis of total $\mathrm{CD}^{+} \mathrm{T}$ cells isolated from PMBCs from males or females. Dot plots of CD3 and CD8 expression in propidium iodide (PI $\left.^{-}\right) \mathrm{CD}^{+} \mathrm{PMBCs}^{-}$ from males or females are shown. (B) Number of $\mathrm{CD}^{+} \mathrm{CD}^{+} \mathrm{T}$ cells in PMBCs from males or females before and after vaccination. (C) Flow cytometric analysis of $\mathrm{CD}^{+} \mathrm{CD}^{+} \mathrm{COVID}-19$-pentamer ${ }^{+} \mathrm{T}$ cells isolated from PMBCs from males or females after vaccination. Plots of CD8 and COVID-19-pentamer expression in $\mathrm{PI}^{-} \mathrm{CD}^{+}$ PMBCs from males or females are shown.

(D) Number of $\mathrm{CD}^{+} \mathrm{CD}^{+} \mathrm{COVID}-19$-pentamer ${ }^{+} \mathrm{T}$ cells among PMBCs from males and females after vaccination. (E) Scatter plots of antibody titer and COVID-19pentamer ${ }^{+} \mathrm{CD}^{+} \mathrm{T}$ cell frequency in males and females after vaccination. (F) Scatter plots of antibody titer and cell number of COVID-19-pentamer ${ }^{+} \mathrm{CD} 8^{+} \mathrm{T}$ cells in males and females after vaccination. ${ }^{* *}, \mathrm{p}<0.01 ;$ n.s., not significant.

\section{Figure 3}

(A) Flow cytometric analysis of $\mathrm{CD}^{+} \mathrm{CD}^{+} \mathrm{CCR} 7^{+} \mathrm{CD} 45 \mathrm{RA}^{+}$(naïve: $\mathrm{Nv}$ ), $\mathrm{CCR}^{+}{ }^{+} \mathrm{CD} 45 \mathrm{RA}^{-}$(central memory: $\left.\mathrm{CM}\right), \mathrm{CCR}^{-} \mathrm{CD}^{-}$45A- (effector memory: EM) and $\mathrm{CCR}^{-}{ }^{-} \mathrm{CD} 45 \mathrm{RA}^{+}$(terminal effector memory: EMRA) T cells among CD8+ $\mathrm{T}$ cells from male or female before or after vaccination. Dot plots of CD45RA and CCR7 expression in $\mathrm{PI}^{-} \mathrm{CD}^{+} \mathrm{CD}^{+}$PMBCs are shown. (B) Frequnecy of Nv, CM, EM and EMRA CD8 ${ }^{+}$ $T$ cells among PMBCs from males or females before or after vaccination. (C) Flow cytometric analysis of $\mathrm{CD}^{+} \mathrm{CD}^{+} \mathrm{COVID}-19$-pentamer ${ }^{+} \mathrm{Nv}, \mathrm{CM}, \mathrm{EM}$ and EMRA T cells among PMBCs from males or females after vaccination. Dot plots of CD45RA and CCR7 expression in $\mathrm{PI}^{-} \mathrm{CD} 3^{+} \mathrm{CD} 8{ }^{+} \mathrm{COVID}-19-$ pentamer $^{+} \mathrm{PMBC}$ are shown. (D) Frequency of Nv, CM, EM and EMRA COVID-19-pentamer ${ }^{+} \mathrm{CD}^{+} \mathrm{T}$ cells among PMBCs from males or females after vaccination. (E) Flow cytometric analysis of 


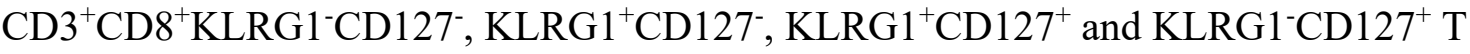
cells among $\mathrm{PMBCs}$ from males or females before or after vaccination. Dot plots of KLRG1 and CD127 expression in $\mathrm{PI}^{-} \mathrm{CD}^{+}{ }^{+} \mathrm{CD} 8^{+} \mathrm{PMBC}$ are shown. (F) Frequency of

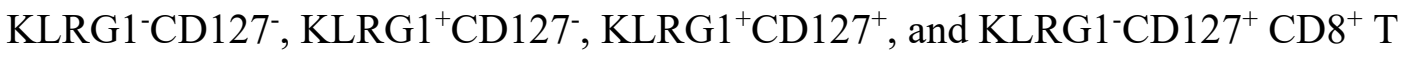
cells among PMBCs from males or females before or after vaccination. (G) Flow cytometric analysis of $\mathrm{CD}^{+}{ }^{+} \mathrm{CD} 8^{+} \mathrm{COVID}-19$-pentamer ${ }^{+} \mathrm{KLRG1}^{-} \mathrm{CD} 127^{-}$, KLRG $1^{+} \mathrm{CD} 127^{-}, \mathrm{KLRG}^{+} \mathrm{CD} 127^{+}$and $\mathrm{KLRG1}{ }^{-\mathrm{CD}} 127^{+} \mathrm{T}$ cells among PMBCs from males or females after vaccination. Dot plots of KLRG1 and CD127 expression in $\mathrm{PI}^{-}$ $\mathrm{CD}^{+}{ }^{+} \mathrm{CD} 8^{+}$PMBCs are shown. (H) Frequency of KLRG1 ${ }^{-} \mathrm{CD} 127^{-}, \mathrm{KLRG}^{+} \mathrm{CD} 127^{-}$, $\mathrm{KLRG}^{+} \mathrm{CD} 127^{+}$, and $\mathrm{KLRG1}^{-\mathrm{CD}} 127^{+} \mathrm{COVID}-19$-pentamer ${ }^{+} \mathrm{CD} 8^{+} \mathrm{T}$ cells among PMBCs from males or females after vaccination. (I) Scatter plots of the number of pentamer ${ }^{+} \mathrm{KLRG} 1^{-} \mathrm{CD} 127^{+} \mathrm{CD} 8^{+} \mathrm{T}$ cells and the percentage of COVID-19pentamer ${ }^{+} \mathrm{CD} 8^{+} \mathrm{T}$ cells after vaccination. ${ }^{*}, \mathrm{p}<0.05$; n.s., not significant.

\section{Figure 4}

(A) Flow cytometric analysis of $\mathrm{CD} 3^{+} \mathrm{CD} 8^{+} \mathrm{PD}-1^{+}$or $\mathrm{CD} 28^{+} \mathrm{T}$ cells among PMBCs from males or females after vaccination. Dot plots of CD28 and PD-1 expression in $\mathrm{PI}^{-}$ $\mathrm{CD}^{+} \mathrm{CD}^{+}$PMBCs are shown. (B) Frequency of $\mathrm{CD} 28^{+}$or $\mathrm{PD}-1^{+} \mathrm{CD} 8^{+} \mathrm{T}$ cells among PMBCs from males or females after vaccination. (C) Flow cytometric analysis of $\mathrm{CD}^{+}{ }^{+} \mathrm{CD} 8^{+} \mathrm{COVID}-19$-pentamer ${ }^{+} \mathrm{PD}-1^{+}$or $\mathrm{CD} 28^{+} \mathrm{T}$ cells among PMBCs from males or females after vaccination. Dot plots of CD28 and PD-1 expression in $\mathrm{PI}^{-}$ $\mathrm{CD}^{+} \mathrm{CD}^{+}$COVID-19-pentamer ${ }^{+}$PMBCs are shown. (D) Frequency of CD $28^{+}$or PD$1^{+}$COVID-19-pentamer ${ }^{+} \mathrm{CD}^{+} \mathrm{T}$ cells among PMBCs from males or females after vaccination. (E) Flow cytometric analysis of $\mathrm{CD}^{+} \mathrm{CD}^{+} \mathrm{CD} 38^{-} \mathrm{HLA}^{-\mathrm{DR}}{ }^{-}, \mathrm{CD} 38^{+} \mathrm{HLA}-$ $\mathrm{DR}^{-}, \mathrm{CD} 38^{+} \mathrm{HLA}^{-\mathrm{DR}^{+} \text {and CD38-HLA-DR }}{ }^{+} \mathrm{T}$ cells among PMBCs from males or females after vaccination. Dot plots of CD38 and HLA-DR expression in $\mathrm{PI}^{-} \mathrm{CD}^{+}{ }^{+} \mathrm{CD} 8^{+}$ PMBCs are shown. (F) Frequency of CD38-HLA-DR', CD38 ${ }^{+}{ }^{-}{ }^{-}$LA-DR', CD38 $8^{+}$HLA$\mathrm{DR}^{+}$and $\mathrm{CD} 38^{-} \mathrm{HLA}-\mathrm{DR}^{+} \mathrm{CD}^{+} \mathrm{T}$ cells among PMBCs from males or females after vaccination. (G) Flow cytometric analysis of $\mathrm{CD} 3{ }^{+} \mathrm{CD} 8{ }^{+} \mathrm{COVID}-19-$ pentamer ${ }^{+} \mathrm{CD} 38$

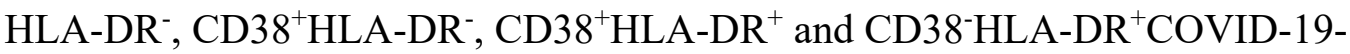
pentamer ${ }^{+} \mathrm{CD} 8^{+} \mathrm{T}$ cells among $\mathrm{PMBC}$ s from males or females after vaccination. Dot plots of CD38 and HLA-DR expression in $\mathrm{PI}^{-} \mathrm{CD} 3{ }^{+} \mathrm{CD}^{+} \mathrm{COVID}-19$-pentamer ${ }^{+} \mathrm{PMBCs}$ are shown. (H) Frequency of CD38-HLA-DR', CD38 ${ }^{+}$HLA-DR-, CD38 ${ }^{+}{ }^{-}{ }^{-}{ }^{-}{ }^{-}-\mathrm{DR}^{+}$and CD38-HLA-DR ${ }^{+}$COVID-19-pentamer ${ }^{+} \mathrm{CD}^{+}$cells among PMBCs from males or females after vaccination. (I) Scatter plots of the number of pentamer ${ }^{+}$CD38-HLA- 
$\mathrm{DR}^{+} \mathrm{CD} 8^{+} \mathrm{T}$ cells and percent of COVID-19-pentamer ${ }^{+} \mathrm{CD} 8^{+} \mathrm{T}$ cells after vaccination. *, $\mathrm{p}<0.05$; n.s., not significant.

\section{Figure 5}

(A) Flow cytometric analysis of CD107a ${ }^{+} \mathrm{COVID}-19$-pentamer ${ }^{+} \mathrm{CD} 8^{+} \mathrm{T}$ cells among PMBCs from males and females cultured for 0 days and 14 days. Dot plots of CD8 and CD107a expression in $\mathrm{PI}^{-} \mathrm{CD} 3{ }^{+} \mathrm{CD}^{+} \mathrm{COVID}-19$-pentamer ${ }^{+} \mathrm{PMBC}$ are shown. (B) Frequency of CD107a $\mathrm{a}^{+}$COVID-19-pentamer ${ }^{+} \mathrm{CD} 8^{+} \mathrm{T}$ cells among PMBCs from males and females cultured for 0 days and 14 days. (C) LEGENDplex ${ }^{\mathrm{TM}}$ multianalyte flow assay of IL-4, IL-6, IL-10, IL-17A, sFas, sFas-L, perforin, TNF- $\alpha$, IFN $\gamma$, granulysin, granzyme A and granzyme B from PMBCs from males and females cultured for 0 days and 14 days. ${ }^{*}, \mathrm{p}<0.05$; n.s., not significant. 


\section{Figure1}

A

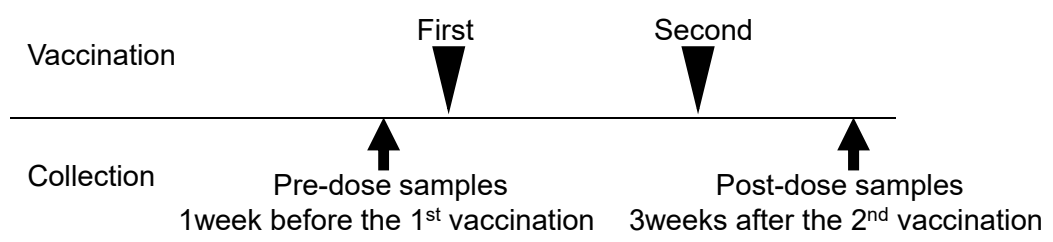

C

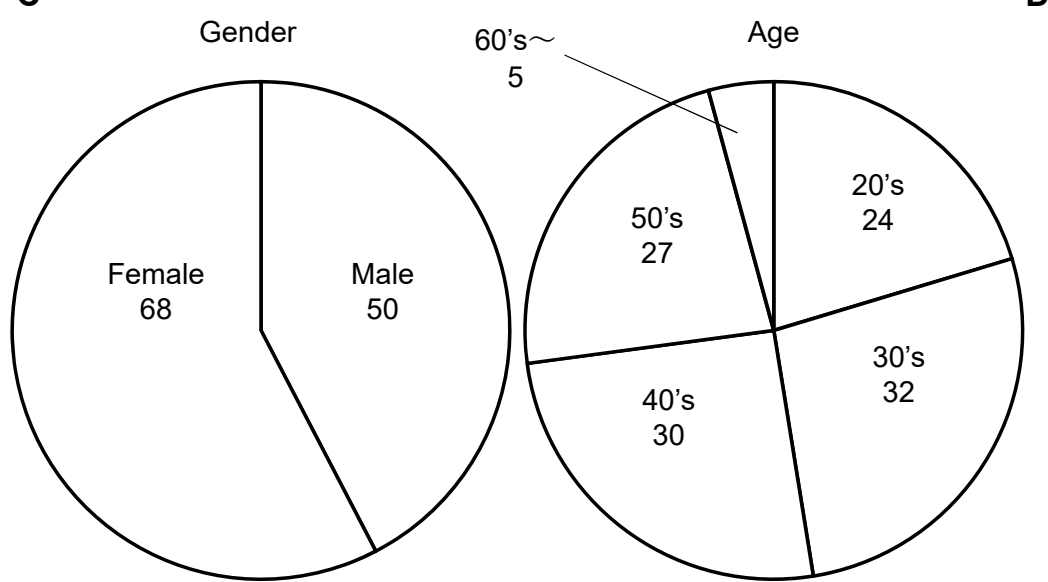

B

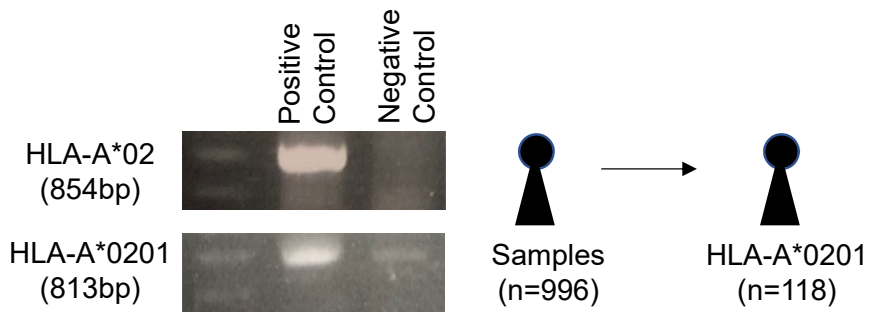

D

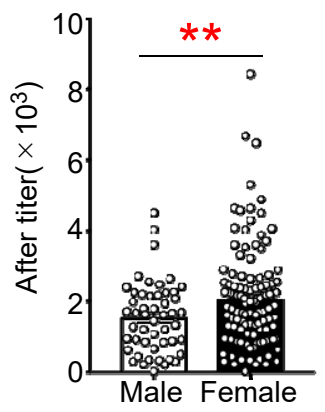

E

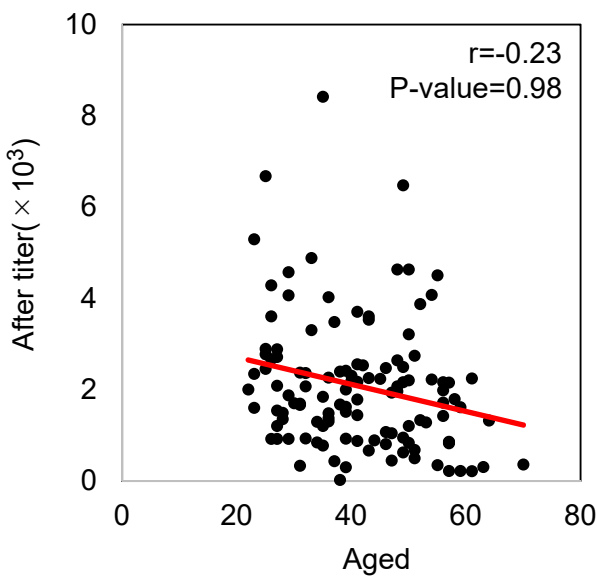




\section{Figure 2}

A

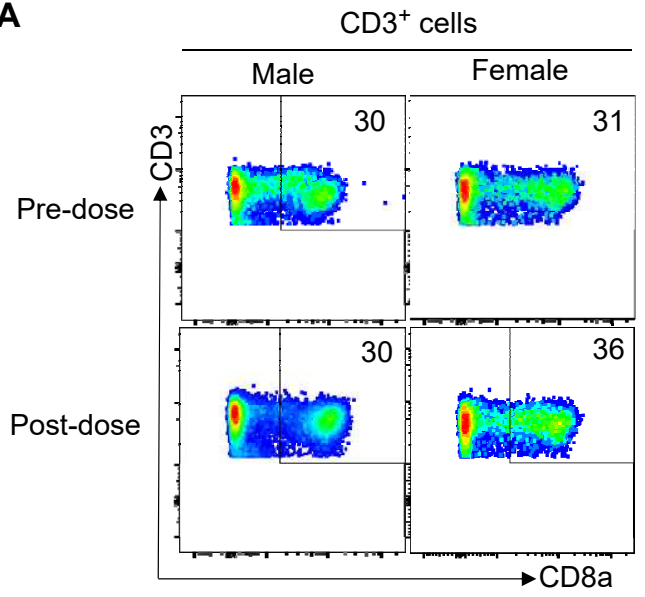

C

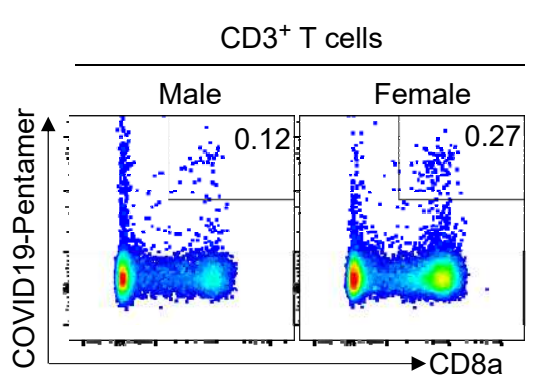

E

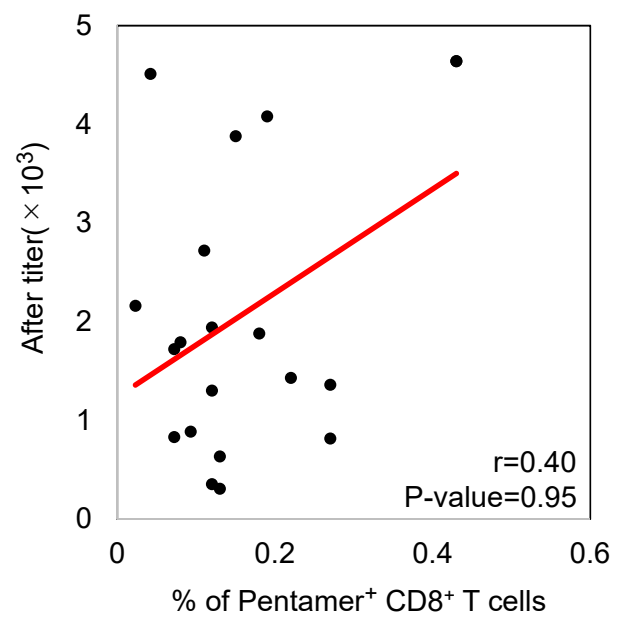

B

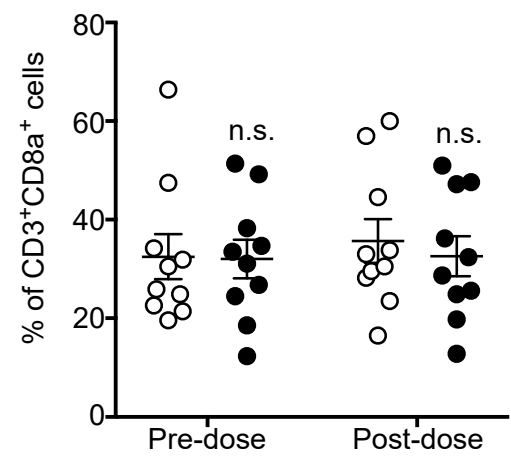

D

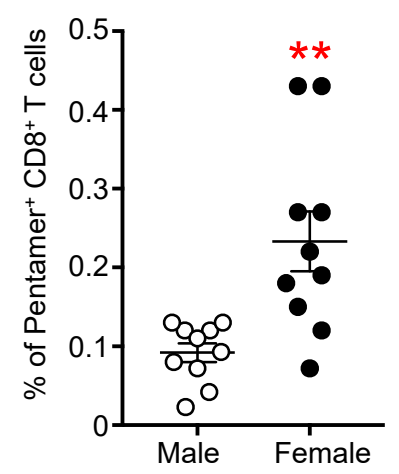

F

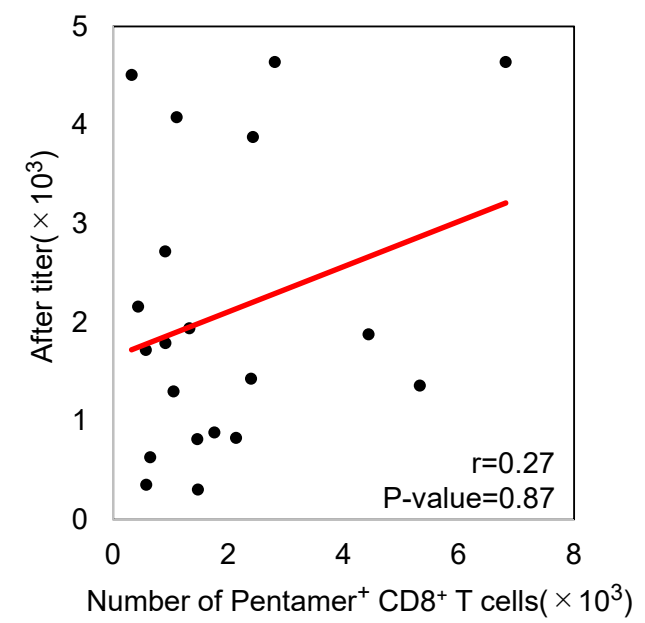




\section{Figure 3}

A

Total $\mathrm{CD}^{+} \mathrm{T}$ cells
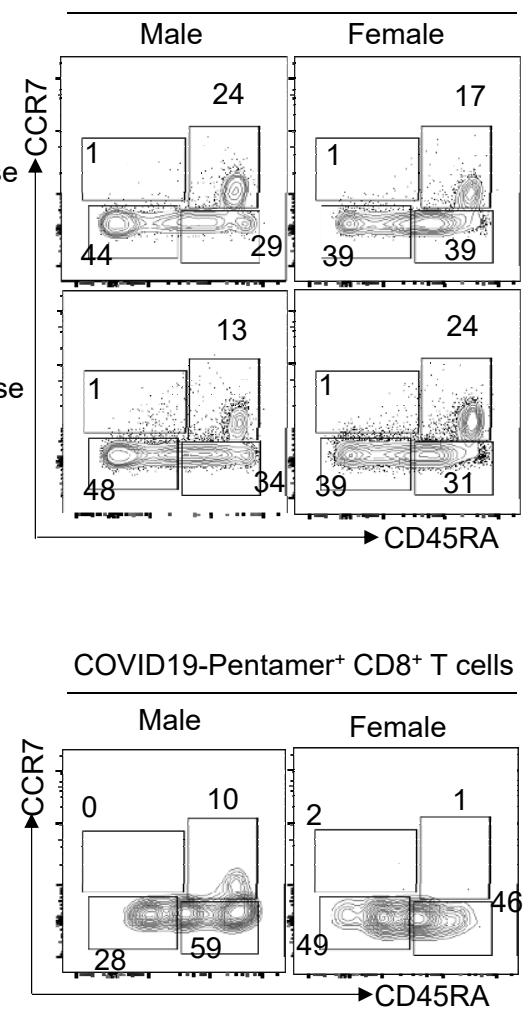

E

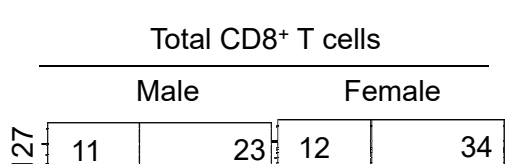

COVID19-Pentamer ${ }^{+} \mathrm{CD} 8^{+} \mathrm{T}$ cells

\section{列}

Pre-dose

Post-dose

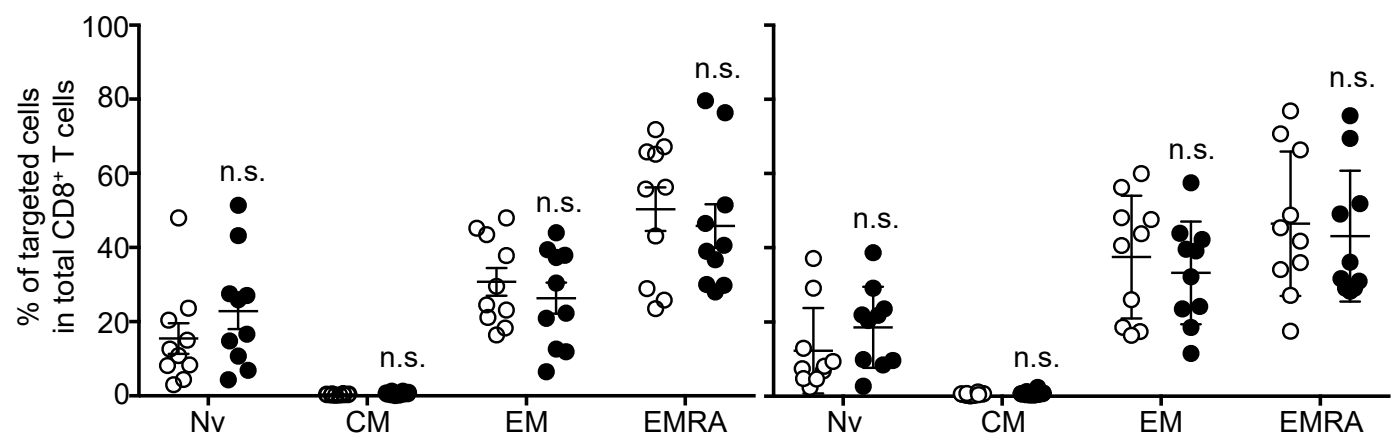

D

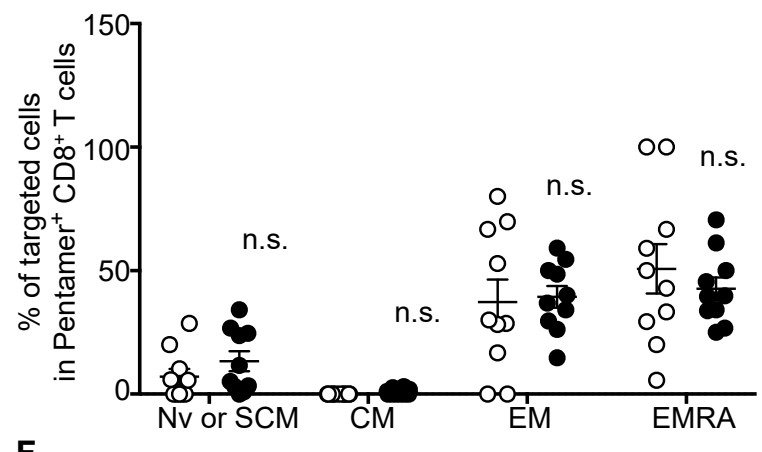

$\mathbf{F}$

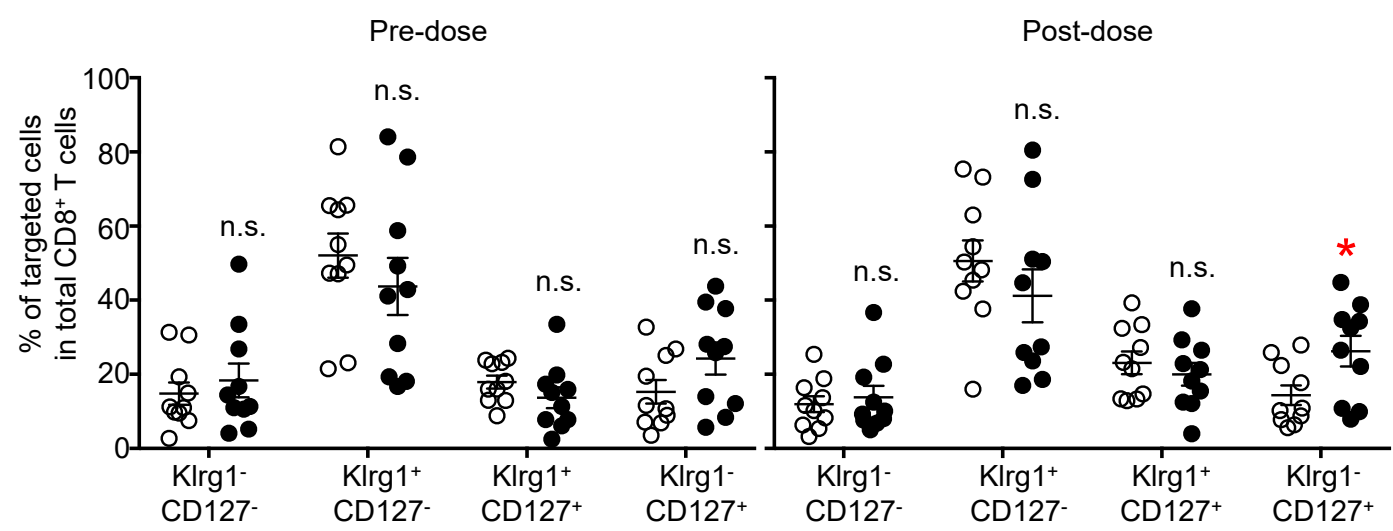

H
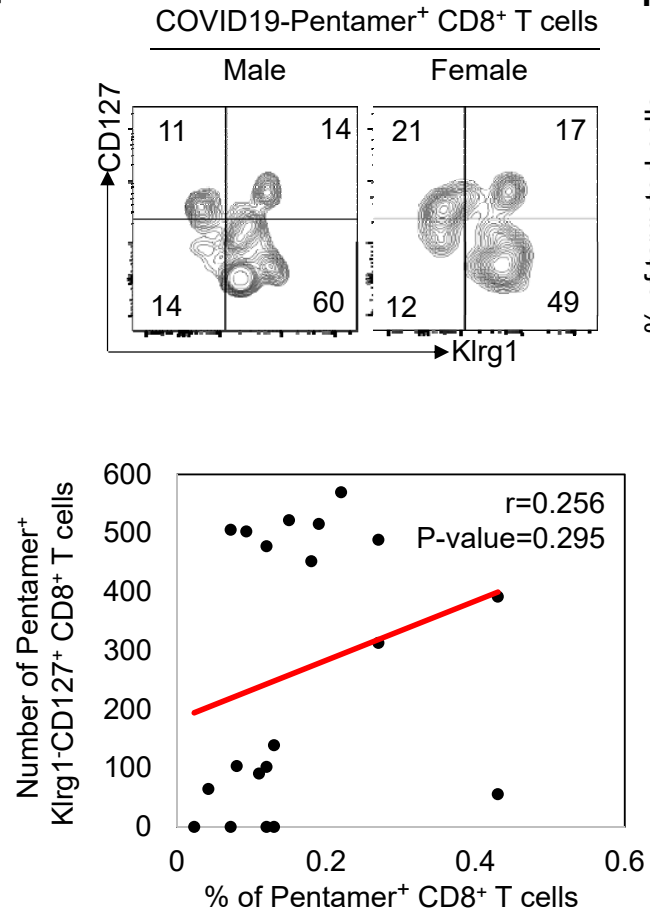


\section{Figure 5}

A

COVID19-Pentamer ${ }^{+} \mathrm{CD}^{+} \mathrm{T}$ cells

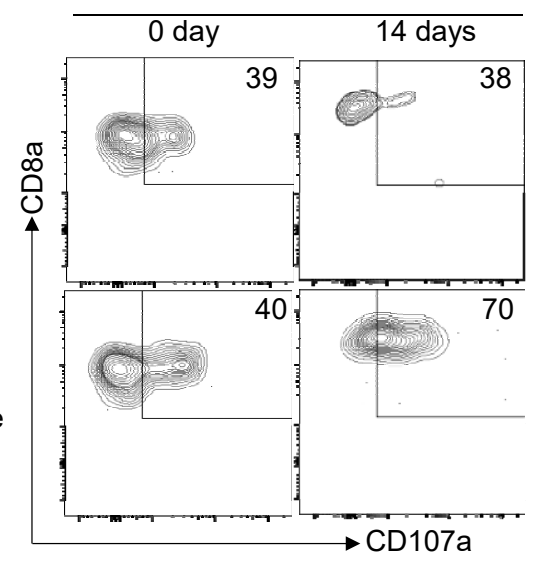

C

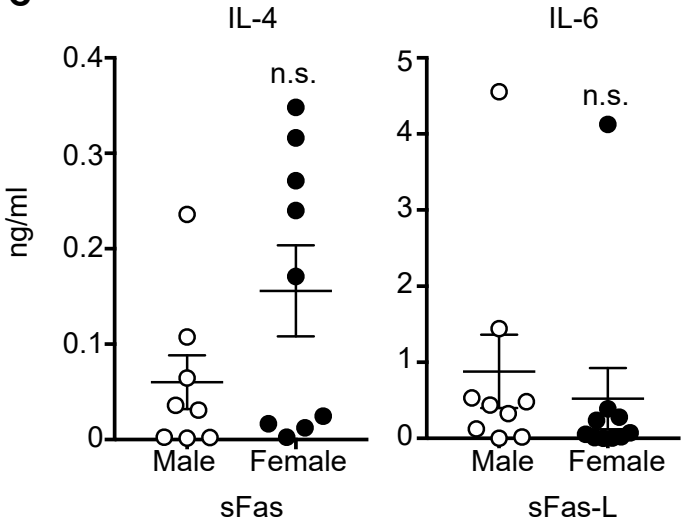

B

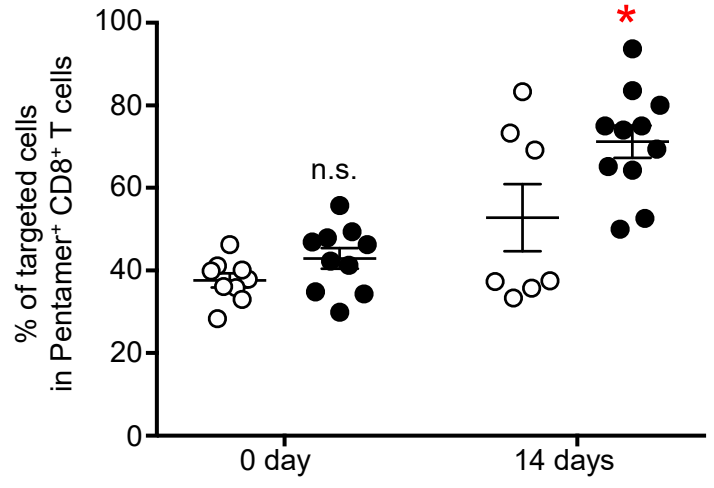

IL-10

IL-17A
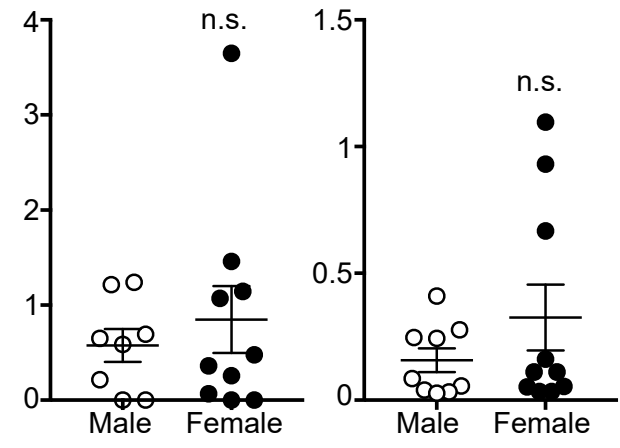

TNF- $\alpha$
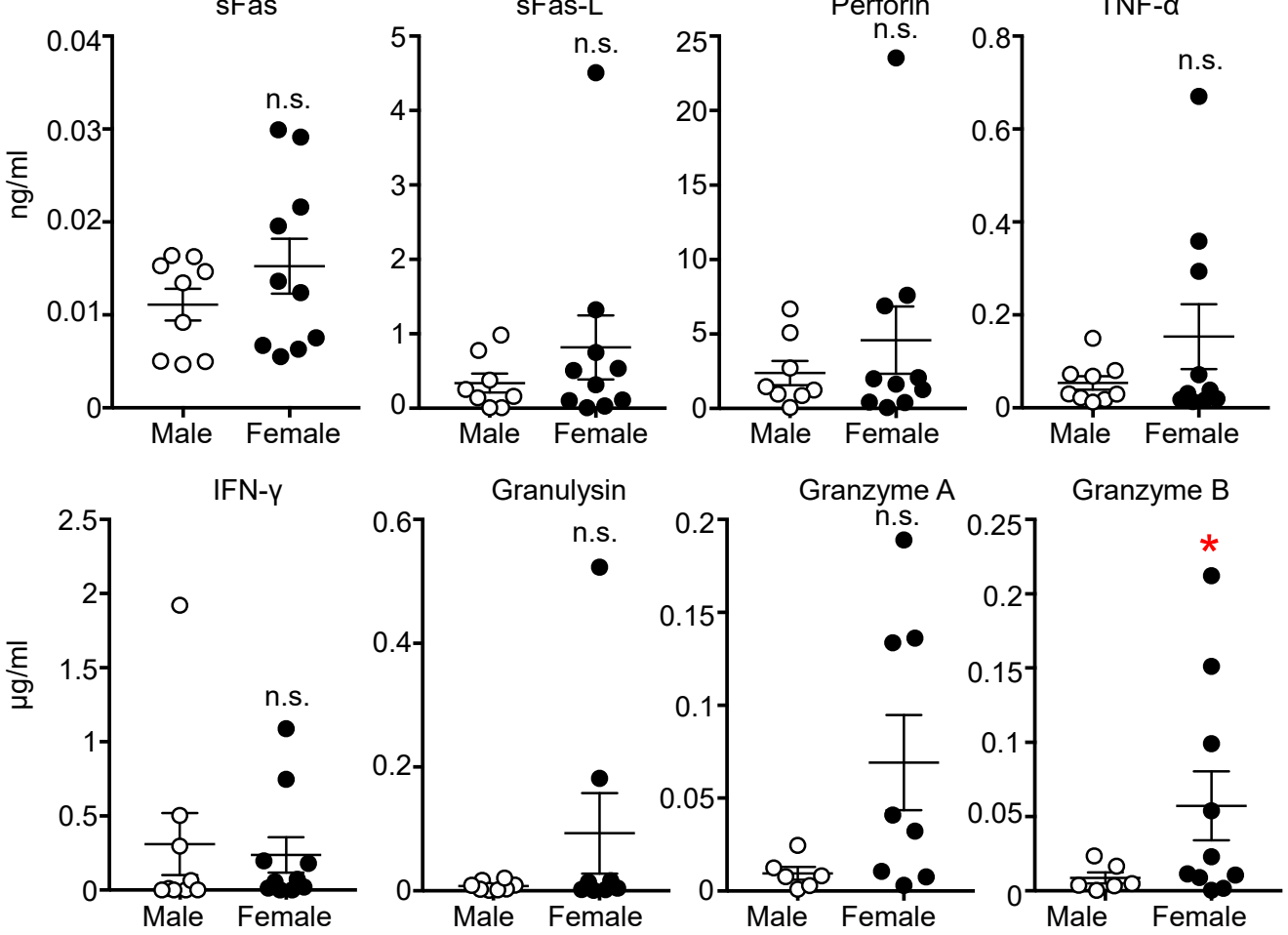